\title{
The cool Galactic R Coronae Borealis variable DY Persei
}

\author{
L. Začs ${ }^{1}$, W. P. Chen ${ }^{2}$, O. Alksnis ${ }^{1}$, D. Kinoshita ${ }^{2}$, F. A. Musaev ${ }^{3,4,5}$, T. Brice ${ }^{6}$, K. Sanchawala ${ }^{2}$, \\ H. T. Lee ${ }^{2}$, and C. W. Chen ${ }^{2}$
}

${ }^{1}$ Institute of Atomic Physics and Spectroscopy, University of Latvia, Raiņa bulvāris 19, 1586 Rīga, Latvia e-mail: zacs@latnet.lv

2 Graduate Institute of Astronomy, National Central University, Chung-Li 32054, ROC, Taiwan e-mail: wchen@astro.ncu.edu.tw

3 Special Astrophysical Observatory and Isaac Newton Institute of Chile, SAO Branch, Nizhnij Arkhyz 369167, Russia e-mail: faig@sao.ru

${ }^{4}$ ICAMER, National Academy of Sciences of Ukraine, 361605 Peak Terskol, Kabardino-Balkaria, Russia

5 Shamakhy Astrophysical Observatory, National Academy of Sciences of Azerbaijan, Azerbaijan

6 1.State Gimnazium of Riga, Raiņa bulvāris 8, 1050 Rīga, Latvia e-mail: tamara@r1g.edu.lv

Received 1 March 2005 / Accepted 28 April 2005

\begin{abstract}
Results of first CCD photometry during the recent deep light decline, and high-resolution spectroscopy, are presented for DY Persei. The spectra show variable blueshifted features in the sodium D lines. The C I lines are strong whereas neutroncapture elements are not enhanced. The isotopic ${ }^{13} \mathrm{CN}(2,0)$ lines relative to ${ }^{12} \mathrm{CN}$ are of similar strength with those for the carbon star U Hya. All these confirm the RCB nature of DY Per and the existence of ejected clouds. At least two clouds are revealed at -197.3 and $-143.0 \mathrm{~km} \mathrm{~s}^{-1}$. A star was detected about 0.4 to the west and $2 . .5$ to the north from DY Per. This anonymous companion, with observed colour indices $(B-V)=0.68$ and $(V-R) \simeq 1.1$, may be a foreground star.
\end{abstract}

Key words. stars: carbon - stars: winds, outflows - stars: fundamental parameters - stars: circumstellar matter stars: individual: DY Per

\section{Introduction}

The prototype of R Coronae Borealis (RCB) stars was observed for the first time more than 200 years ago. However, after two centuries of investigations many aspects of the $\mathrm{R} \mathrm{CrB}$ phenomenon remain mysterious (for a general review, see Clayton 1996). The atmospheres of RCB stars are extremely hydrogendeficient but rich in carbon and nitrogen (Asplund et al. 2000, and references therein). RCBs seem to be of low mass yet high luminosity. At irregular intervals they manufacture thick dust clouds which can completely obscure the photosphere of the star. During these dust formation episodes the brightness of the star can decrease by 5 to $8 \mathrm{mag}$ in a few weeks and characteristic emission lines are seen in the spectrum. Recently several bright, very large circumstellar dust clouds were detected in RCB variable RY Sgr (de Laverny \& Mékarnia 2004). These enigmatic stars seem to be born-again giants, formed either through a final He-shell flash in post-AGB stars or through a merger of two white dwarfs. No RCB star is known to be a binary.

DY Per (CGCS 372) was suspected to be an RCB star based on the photometry by Alksnis (1994). Subsequent photometric monitoring revealed a number of sudden light declines typical for RCB variables superimposed on the 792-day cycle of long-period light variations (Alksnis et al. 2002). However, the temperature of this star is much lower than average for other RCB stars. Moreover, the luminosity of DY Per was suspected to be lower than usually for RCBs. Keenan \& Barnbaum (1997) estimated $T_{\text {eff }} \simeq 3500 \mathrm{~K}$ using the standard criteria of spectral classification and a giant luminosity. DY Per was classified as R8 (Hardorp et al. 1973), C 4,5 (Yamashita 1975), C 5,4p (Dean 1976) or C-Hd 4.5 C 6 (Keenan \& Barnbaum 1997). Alcock et al. (2001) revealed four stars similar to the Galactic variable DY Per among eight RCBs discovered in the Large Magellanic Cloud (LMC). They found for RCBs in the LMC a range of absolute magnitudes $M_{V}$ from -2.5 to -5 mag and a temperature $-M_{V}$ relationship. The warm RCBs are brighter than the cool one. Therefore DY Per remains an intriguing object with unknown evolutionary status. Here we present the first results of CCD photometry during the recent deep light decline (see Alksnis 2004), and some results of high-resolution spectroscopy for this unique star.

\section{Observations and data reduction}

The imaging photometry of DY Per was taken with the Lulin 1-m telescope (LOT), equipped with a Princeton Instruments PI 1300B CCD camera. The $\mathrm{f} / 8$ optics of the telescope 
gives 0.516 per pixel and an FOV about $11^{\prime}$ on the focal plane. DY Per was monitored in the $V$ band from early October 2004 through late January 2005, for which differential photometry is available. Typical seeing was about $2^{\prime \prime}$. On several nights, in particular on 11 November 2004 for which the sky conditions were good, a complete set of $B V R I$ data were acquired and calibrations of standard stars and extinction were performed. Exposure times ranged from $20 \mathrm{~s}$ in the $I$ and $R$ bands, $90 \mathrm{~s}$ in the $V$ band, to $300 \mathrm{~s}$ in the $B$ band. The Landolt standards, SA92 \#245, 248, 249, and 250, were used for flux calibration, with a typical internal photometric accuracy better than $\sim 0.01 \mathrm{mag}$. Images were processed by standard procedures of bias and dark subtraction and flat fielding with the IRAF package.

High-resolution spectra for DY Per and the comparison star U Hya were obtained with the coude échelle spectrometer MAESTRO fed by the $2 \mathrm{~m}$ telescope at the Observatory on the Terskol Peak in Northern Caucasus equipped with a CCD detector (Musaev et al. 1999) with a resolving power of $\sim 45000$. A total exposure time of $7200 \mathrm{~s}$ was made for DY Per on 18 November 2002. The spectrum covered from 3600 to $10200 \AA$ in 85 wavelength bands overlapping shortward of $\mathrm{H}_{\alpha}$. Each region spanned from 50 to $140 \AA$. Because of the energy distribution in carbon stars the $S / N$ ratio shortward of $4800 \AA$ is very low. In addition, for comparison purposes one high-resolution spectrum of similar resolution was adopted from Barnbaum (1994) to reveal possible changes in spectra between two seasons of observations. The reduction of spectra was performed with the standard DECH20T routines.

\section{Analysis}

\subsection{Photometry}

Images observed on 11 November 2004 during the deep light decline revealed a nearby star about 0. . 4 to the west and 2.5 to the north from DY Per. Figure 1 shows the images in the $B$ and $I$ bands. Individual fluxes of DY Per and the anonymous star were measured using the IRAF/DAOPHOT for PSF photometry. The carbon star DY Per is much redder than the companion so a clear separation is seen toward shorter wavelengths. The magnitudes for DY Per itself were found to be $m_{B} \sim 17.89 \mathrm{mag}, m_{V} \sim 15.68 \mathrm{mag}$, and $m_{R} \sim 13.89 \mathrm{mag}$, respectively. The anonymous star had $m_{B} \sim 17.48 \mathrm{mag}, m_{V} \sim$ $16.80 \mathrm{mag}$, and $m_{R} \sim 15.66 \mathrm{mag}$, respectively. At longer wavelengths, DY Per outshines the companion. In the $I$ band, the pair could no longer be resolved, and the combined flux, predominantly from DY Per, was $m_{I} \sim 11.85 \mathrm{mag}$.

The anonymous star, judging from its observed colours indices $(B-V)=0.68$ and $(V-R) \simeq 1.1$, is likely a foreground star so not physically related to DY Per. With no prior knowledge of the existence of the anonymous star, previous photometric measurements of DY Per, in particular toward short wavelengths, conceivably could have been influenced.

For the photometry obtained on other nights only the combined fluxes of DY Per and its companion were estimated (see Table 1). DY Per appeared to have brightened between midNovember and mid-December of 2004.
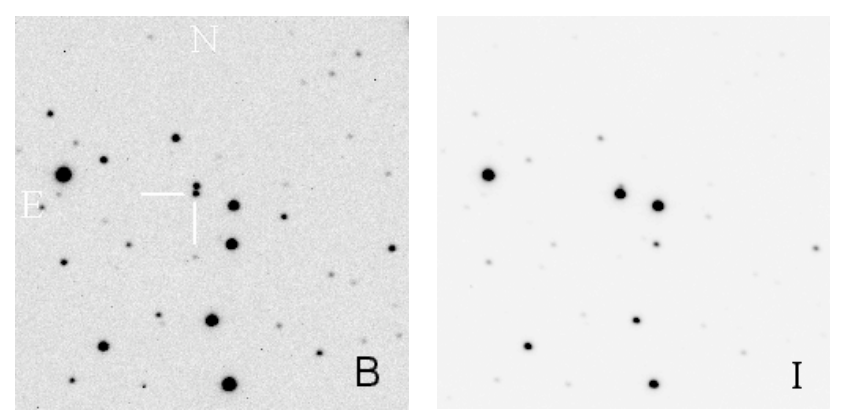

Fig. 1. The star field around DY Per (marked) in the $B$ (left) and $I$ (right) bands observed with the Lulin 1-m telescope. An anonymous star is seen to the north of DY Per.

Table 1. The combined visual magnitude of DY Per and the nearby star.

\begin{tabular}{rrc}
\hline \hline Date & JD & $\begin{array}{c}V \\
\text { mag }\end{array}$ \\
\hline 2004 Oct. 4 & 2453282.5 & 15.48 \\
28 & 306.5 & 15.26 \\
Nov. 1 & 310.5 & 15.28 \\
7 & 316.5 & 15.34 \\
11 & 320.5 & 15.35 \\
Dec. 17 & 356.5 & 14.83 \\
2005 Jan. 21 & 391.5 & 14.64 \\
24 & 394.5 & 14.55 \\
29 & 399.5 & 14.46 \\
\hline
\end{tabular}

\subsection{Radial velocity}

The radial velocity (RV) for DY Per was measured from the high-resolution MAESTRO spectrum (JD 2452 597.5) using about 30 relatively clean and symmetric atomic absorption lines selected over the whole spectral region. In addition about 60 uncontaminated $\mathrm{CN}$ lines were selected for $R V$ measurements. No significant difference was found in the velocity derived from atomic or molecular lines. The heliocentric correction was calculated to be $-0.5 \mathrm{~km} \mathrm{~s}^{-1}$. Thus the mean heliocentric stellar $R V_{\odot}$ was found to be $-43.7 \mathrm{~km} \mathrm{~s}^{-1}$ with a standard deviation of the mean value of $0.4 \mathrm{~km} \mathrm{~s}^{-1}$.

Radial velocity for DY Per using the same (common) lines was estimated also in the spectrum observed at Lick Observatory (JD 2448141.5 ),$R V_{\odot}=-47.2 \mathrm{~km} \mathrm{~s}^{-1}$, which is close to that (-46.8) obtained by Barnbaum (1992) using the cross correlation technique against the template of a standard carbon star. The difference between the stellar radial velocities derived for two seasons seems to be significant. We note that Dean (1976) reported $R V_{\odot}=-39 \pm 5 \mathrm{~km} \mathrm{~s}^{-1}$ using medium-resolution spectra. The change could be the result of pulsations in this SRb variable. Barnbaum (1992) using cross-correlation analysis found the mean radial velocity to vary about $3 \mathrm{~km} \mathrm{~s}^{-1}$ for SR and Lb variable carbon stars. Spectroscopic monitoring, preferably with simultaneous photometric measurements, is highly desirable to clarify the nature of RV variations in DY Per. 


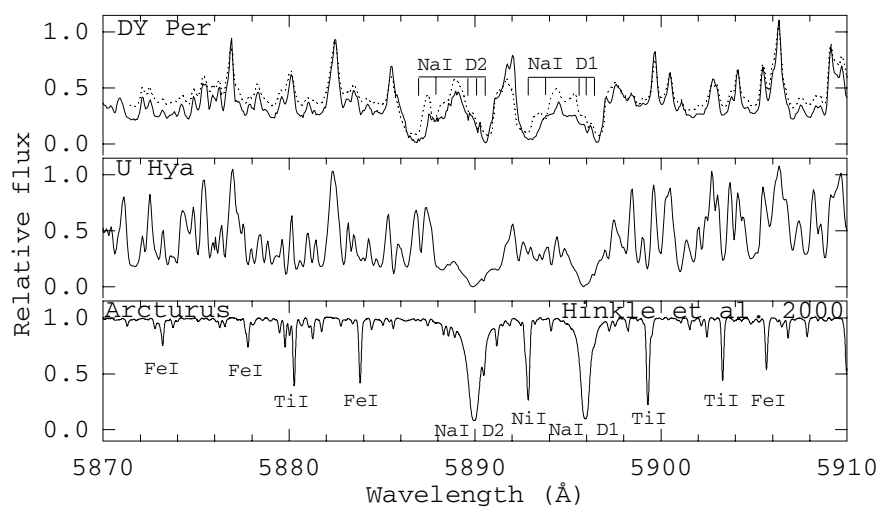

Fig. 2. The observed spectra of DY Per $(C 4.5,6)$ around the Na I D12 lines for two different seasons, November 2002 (solid line) and September 1990 (dotted line; Keenan \& Barnbaum 1997), both near light maxima. Also shown are the spectra for a typical carbon star U Hya (C 6,5) and the standard star Arcturus (K $1.5 \mathrm{III})$. All spectra have been shifted in wavelengths to correct for the stellar radial velocities. The resolved five Na I components are indicated by ticks.

The resulting rectified spectrum for the region around NaID12 lines along with those for the typical carbon star $\mathrm{U}$ Hya $(\mathrm{C} 6,5)$ is presented in Fig. 2; also shown is the spectrum for the standard K 1.5 giant Arcturus (Hinkle et al. 2000). Although the spectrum of DY Per is very crowded the spectral features seen in both spectra (seasons) are almost identical. Some discrepancies in the relative intensities seem to be mainly due to uncertainties in the continuum definition. However, significant changes are seen in the sodium lines. At least five components are resolved in each of the spectra. Averaged D1 and D2 velocities of these components on 2002 (phase 0.77) and 1990 (phase 0.15) are (-197.3, -143.0, -57.0, $-36.7,-12.8) \mathrm{km} \mathrm{s}^{-1}$ and $(-214.4,-157.8,-56.8,-36.6$, $-10.4) \mathrm{km} \mathrm{s}^{-1}$, respectively. The phases are calculated using Max $=2438$ 521+792E (Alksnis 1994). Each measured radial velocity refers to the line core, and for an individual line is accurate to about $1.5 \mathrm{~km} \mathrm{~s}^{-1}$. It is evident that the two most blueshifted components display changes both in the radial velocity and the shape, thus lending support to the assertion (see Keenan \& Barnbaum 1997) that the most blueshifted component would be formed in the cloud of dust and gas ejected by DY Per. However, the interstellar origin of the component at $-157.8 \mathrm{~km} \mathrm{~s}^{-1}$ was overruled due to significant changes in the profile and velocity. Thus at least two clouds ejected by star are detected. Three components near the stellar velocity of DY Per seem to be non-variable so originate from the photosphere and interstellar medium. The existence of ever-changing thick dust clouds near the surface of the RCB star UW Centauri was discovered by Clayton et al. (1999). De Laverny \& Mékarnia (2004) reported the first direct detection of heterogeneities in the circumstellar envelope of the RCB variable RY Sgr. Thus our spectroscopy support the $\mathrm{RCB}$ nature of DY Per.

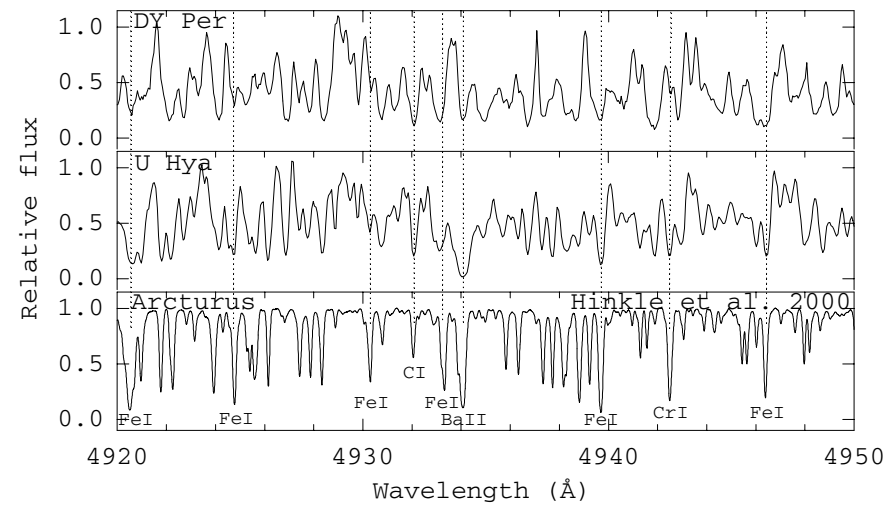

Fig. 3. The observed spectra around the Ba II line at $4934 \AA$ where contamination by molecular lines is relatively low. It is seen that barium is not enhanced in the atmosphere of DY Per. The strong C I line at $4932 \AA$ is marked.

\subsection{Chemical peculiarities}

The use of traditional method to calculate atmospheric parameters and abundances for DY Per is not possible mainly due to the lack of reliable atmospheric models. Asplund et al. (1997) calculated hydrogen-deficient model atmospheres for typical RCB stars only with effective temperatures higher than $5000 \mathrm{~K}$. Here a few results of qualitative analysis are provided those based mainly on comparison of our high-resolution spectra for DY Per (C 4.5,6), U Hya (C 6,5) and the standard K 1.5 giant Arcturus. In the red spectral region the influence of the nearby star should be negligible, because the spectrum of DY Per was observed near the maximum of light and the companion is much hotter than DY Per itself.

An inspection of the high-resolution spectra of DY Per relative to the normal carbon star $\mathrm{U}$ Hya $([\mathrm{Fe}] \simeq 0.0,[\mathrm{~s} / \mathrm{Fe}] \simeq$ +1 dex, ${ }^{12} \mathrm{C} /{ }^{13} \mathrm{C}=35$; see Abia et al. 2002) shows that a large number of strong $\mathrm{C}_{2}$ and $\mathrm{CN}$ lines dominate over all the analyzed spectrum, blending significantly with the atomic absorption lines. However, a limited number of relatively uncontaminated atomic lines were selected to check chemical peculiarities revealed for typical RCBs. Our analysis shows that the lines of the iron-peak elements are of similar strength or slightly deficient while features due to the atomic carbon are enhanced (see, for example, Fig. 3), in agreement with that for typical RCB stars. The equivalent widths of strong $\mathrm{C}$ I line at $4932 \AA$ in the spectra of DY Per and U Hya are $E W \simeq 500$ and $280 \mathrm{~m} \AA$, respectively. The shape of the spectrum of carbon star determine mainly the absolute abundances of CNO elements and the $\mathrm{C} / \mathrm{O}$ ratio. The extremely complicated spectrum of DY Per (due to carbon bearing molecules) in comparison with that for the carbon star $\mathrm{U}$ Hya support high $\mathrm{C} / \mathrm{O}$ value for DY Per. However, it is evident that neutron-capture elements are not enhanced. For example, the barium line at $4934 \AA$ in the spectrum of DY Per is relatively weak $(E W \simeq 400 \mathrm{~m} \AA$; see Fig. 3) in comparison with that for U Hya $(E W \simeq 1 \AA)$, confirming nearly solar barium abundance. Notice that typical RCB stars show weak hydrogen and strong $\mathrm{C}(\mathrm{N})$ lines; the s-process elements usually are mildly enhanced (Asplund et al. 1997). Relative intensities of spectral features due to 


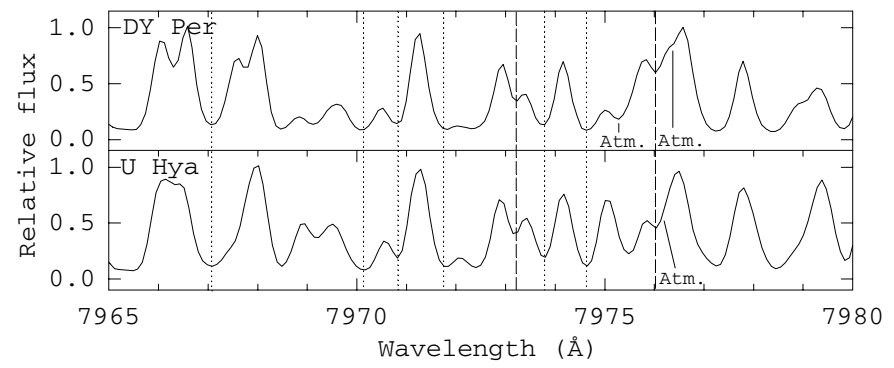

Fig. 4. The spectra of DY Per and U Hya in the spectral region from 7965 to $7977 \AA$. The isotopic ${ }^{13} \mathrm{CN}(2,0)$ lines (dashed line) relative to ${ }^{12} \mathrm{CN}$ (dotted line) are of similar strength with those for the carbon star U Hya with ${ }^{12} \mathrm{C} /{ }^{13} \mathrm{C}=35$.

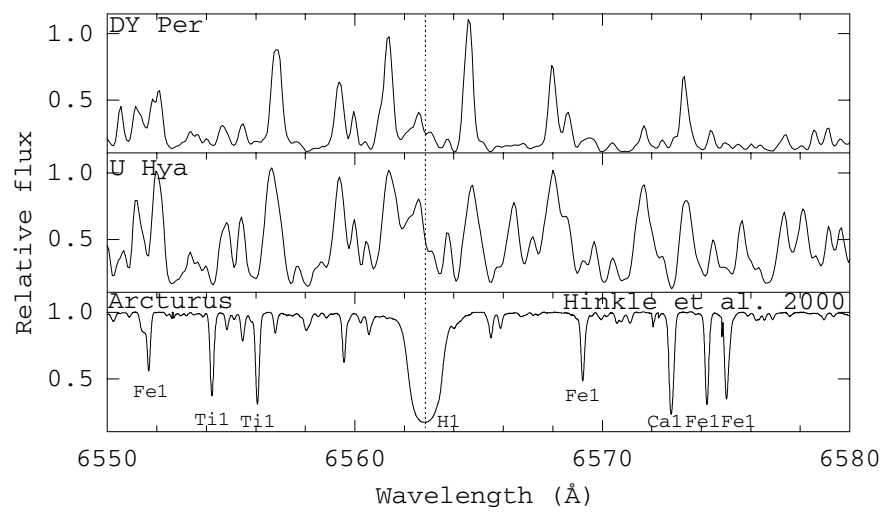

Fig. 5. The observed spectra around the $\mathrm{H}_{\alpha}$ line. The laboratory wavelength of $\mathrm{H}_{\alpha}$ line is marked by the dotted line. The small dip in the spectra of DY Per and U Hya could be the core of $\mathrm{H}_{\alpha}$. In this case hydrogen deficiency in the atmosphere of DY Per is doubtful.

isotopic molecules were inspected using the $\mathrm{C}_{2}$ Swan system band heads near $4740 \AA$ and some lines of $\mathrm{CN}$ red system $(2,0)$ at $7975 \AA$. It is evident that the isotopic ${ }^{13} \mathrm{CN}$ lines relative to ${ }^{12} \mathrm{CN}$ are of similar strength with those for the carbon star U Hya (see Fig. 4). The discrepancy with some previous estimations seems to be due to neglected blends in the used low-resolution spectra. Unfortunately, the regions around $\mathrm{H}_{\alpha}$ (Fig. 5) and $\mathrm{H}_{\beta}$ lines are too crowded to clarify the level of hydrogen deficiency in the atmosphere of DY Per even in the high-resolution spectrum. An inspection of another lines of the Balmer series was not possible due to very low $S / N$ ratio in the blue region of high-resolution spectrum.

\section{Discussion and conclusions}

1. An inspection of the high-resolution spectrum of DY Per observed near the light maximum relative to the normal carbon star U Hya shows that neutron-capture elements are not enhanced while the features due to atomic carbon are strong, in agreement with that for typical RCB stars. The metallicity was found to be nearly solar. The isotopic ${ }^{13} \mathrm{CN}$ lines are not enhanced in the spectrum of DY Per relative to ${ }^{12} \mathrm{CN}$. Unfortunately the spectral regions around $\mathrm{H}_{\alpha}$ and $\mathrm{H}_{\beta}$ are very crowded to clarify the level of hydrogen deficiency in the atmosphere of DY Per.
2. A significant changes are seen in the profiles of sodium $\mathrm{D}$ lines between two seasons of observations. At least five components are resolved. Averaged D1 and D2 heliocentric velocities of these components on 2002 (phase 0.77) and 1990 (phase 0.15$)$ are $(-197.3,-143.0,-57.0$, $-36.7,-12.8) \mathrm{km} \mathrm{s}^{-1}$ and $(-214.4,-157.8,-56.8,-36.6$, $-10.4) \mathrm{km} \mathrm{s}^{-1}$, respectively. Thus two the most blueshifted components display changes both in the radial velocity and shape while three components near the stellar velocity of DY Per seems to be non-variable. The blueshifted components apparently are formed in the clouds of gas and dust ejected by DY Per. The rest revealed components originate in the stellar atmosphere and interstellar medium.

3. A close nearby star was detected about 2". 5 from DY Per using CCD images during the recent deep light decline. The carbon star DY Per itself is much redder than the companion so a clear separation is seen in the $B$ band. At longer wavelengths DY Per starts to outshines the companion. The presence of such companion was not revealed during more than 10 years of the photometric monitoring, however, it was supposed by Alksnis (1994) to interpret the variations of the observed colour indices. This anonymous companion, with observed colour indices $(B-V)=0.68$ and $(V-R) \simeq 1.1$, may be a foreground star.

Acknowledgements. A. Alksnis is acknowledged for initiation of this research and subsequent discussions. The referee, G. C. Clayton, is thanked for his valuable comments on the paper. We are grateful to Y. T. Chen, P. S. Chiang, H. C. Lin, Z. Y. Lin, and M. C. Tsai for their help with the photometric observations. The Mutual Fund of scientific collaboration among Taiwan (ROC), Latvia and Lithuania is thanked for support. WPC acknolweges the grant from NSC/Taiwan NSC932112-M-008-040.

\section{References}

Abia, C., Dominguez, I., Gallino, R., et al. 2002, ApJ, 579, 817

Alcock, C., Allsman, R. A., Alves, D. R., et al. 2001, ApJ, 554, 298

Alksnis, A. 1994, BaltA, 3, 410

Alksnis, A., Larionov, V. M., Larionova, L. V., \& Shenavrin, V. I. 2002, BaltA, 11, 487

Alksnis, A. 2004, IBVS, 5561, 1

Asplund, M., Gustafsson, B., Kiselman, D., \& Eriksson, K. 1997, A\&A, 318, 521

Asplund, M., Gustafsson, B., Lambert, D. L., \& Rao, N. K. 2000, A\&A, 353, 287

Barnbaum, C. 1992, AJ, 104, 1585

Barnbaum, C. 1994, ApJS, 90, 317

Clayton, G. C. 1996, PASP, 108, 225

Clayton, G. C., Kerber, F., Gordon, K. D., et al. 1999, ApJ, 517, L143

Dean, C. A. 1976, AJ, 81, 364

de Laverny, P., \& Mékarnia, D. 2004, A\&A, 428, 13

Hardorp, J., Lubeck, K., \& Stephenson, C. B. 1973, A\&A, 22, 129

Hinkle, K., Wallace, L., Valenti, J., \& Harmer, D. 2000, Visible and Infrared Atlass of the Arcturus Spectrum 3727-9300 A (San Francisco: ASP)

Keenan, P. C., \& Barnbaum, C. 1997, PASP, 109, 969

Musaev, F. A., Galazutdinov, G. A., Sergeev, A. V., Karpov, N. V., \& Podyachev, Yu. V. 1999, Kinematika I Fizika Nebesnyh Tel, 15, 282

Yamashita, Y. 1975, Ann. Tokyo Astron. Obs., 15, 47 